\title{
Analysis of the Efficacy of a Sublingual Bacterial Vaccine in the Prophylaxis of Recurrent Urinary Tract Infection
}

\author{
Pedro Carrión-López ${ }^{a}$ Jesús Martínez-Ruiza

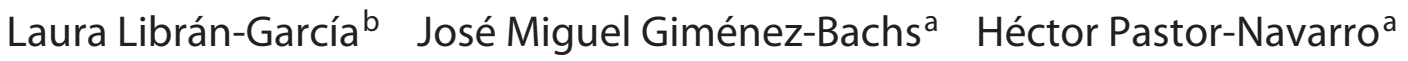 \\ Antonio S. Salinas-Sánchez ${ }^{a}$ \\ ${ }^{a}$ Department of Urology, University Hospital Complex, Albacete, Spain; ${ }^{b}$ Primary Care Management Service, \\ Albacete, Spain
}

\section{Keywords}

Genitourinary tract infection · Immunotherapy · Sublingual administration

\begin{abstract}
Objectives: Recurrent urinary tract infections (R-UTIs) are very common amongst women, and alternatives to antibacterial prophylaxis are necessary. This study evaluates the effectiveness of a sublingual bacterial vaccine for the prophylaxis of R-UTIs. Methods: We conducted a quasi-experimental pretest-posttest study of 166 women diagnosed with R-UTIs. Both before and after the start of treatment with the vaccine, we analysed the total number of R-UTI episodes, the urine culture results, and the type and number of antibiotic packages consumed. Symptoms and urine cultures were evaluated $3,6,9,12,18$, and 24 months after initiating treatment with the vaccine. Results: The mean time of follow-up after vaccination was 1.7 years. After vaccination, there was a $54.6 \%$ reduction in episodes of UTI, and a $56.2 \%$ reduction in positive urine cultures. At 3 months, $74.4 \%$ of the patients had no R-UTI, the rate falling to $68.1 \%$ at 6 months, $52.4 \%$ at 12 months, and $44.5 \%$ at 24 months. The cumulative probability of maintaining negative urine cultures was $76 \%$ at 3
\end{abstract}

months, $37 \%$ at 12 months, and $18 \%$ at 2 years. Conclusions: The use of a sublingual bacterial vaccine for the prophylaxis of R-UTIs in women is an effective treatment that contributes to a reduction in the number of UTI episodes.

(C) 2020 The Author(s)

Published by S. Karger AG, Basel

\section{Introduction}

Urinary tract infections (UTIs) are principally defined as the existence of bacteria in the urinary tract capable of producing functional and/or morphological disorders. Significant bacteriuria associated with infection is defined as the presence of $>100,000 \mathrm{CFU} / \mathrm{mL}$ in a urine culture [1]. Recurrent UTI (R-UTI) is diagnosed when patients manifest 3 or more episodes of UTI in 1 year, or 2 episodes in 6 months [1]. Treatment of R-UTI presents a significant challenge to the professional due to the frequent appearance of symptoms and the high rate of antibiotic resistance as a consequence of antibiotic prophylaxis guidelines aimed at preventing these infections [2]. Antibiotic therapy administered in varying prescription patterns has traditionally been the fundamental strategy for managing R-UTIs. Currently, new "antibiotic-saving" karger@karger.com www.karger.com/uin Karger"

\section{(C) 2020 The Author(s)}

Published by S. Karger AG, Basel

Karger

Open access

This article is licensed under the Creative Commons AttributionNonCommercial-NoDerivatives 4.0 International License (CC BYNC-ND) (http://www.karger.com/Services/OpenAccessLicense). Usage and distribution for commercial purposes as well as any distribution of modified material requires written permission.
Pedro Carrión-López

Department of Urology, University Hospital Complex c/Hellín s/n

ES-02001 Albacete (Spain)

E-Mail pedrocarrion1980@ hotmail.com 
therapies based on natural products are gaining prominence in the prevention of R-UTIs (proanthocyanidin type A [3] or D-mannose [4]) due to their simplicity and safety; however, we currently have insufficient evidence for their efficacy in populations susceptible to UTI [3].

In recent years, the concept of the mucosal immune system has become increasingly important. This involves the immune function shared by, amongst other systems, the mucous membranes of the respiratory tract, the digestive tract, and the genitourinary tract. The genitourinary tract employs an innate and adaptive mucosal immune systemic response to fight against uropathogens. Since immunocytes pass through various mucosa-associated lymphoid tissue (MALT) sites, the activation of lymphocytes at a distant MALT site [5] can facilitate the spread of immunity to other MALT sites. Some studies have found that stimulation of the sublingual mucosa is related to the activation of a broad-spectrum systemic and mucosal immune response in the genitourinary tract. In particular, the response at the site of the bladder mucosa is persistent and highly effective when the sublingual mucosa is stimulated [6]; this is the underlying mechanism of the bacterial vaccine known as Uromune ${ }^{\circledR}$.

The objective of this study was to determine the epidemiological characteristics of our patients with R-UTIs and to evaluate the efficacy of the sublingual bacterial vaccine Uromune ${ }^{\circledR}$ in their treatment. Additionally, we sought to determine which patient characteristics were associated with a greater response to this treatment.

\section{Subjects and Methods}

\section{Study Design}

This is a quasi-experimental, pretest-posttest study of a single group of women diagnosed with R-UTIs who were studied both before being treated with a sublingual bacterial vaccine and for a minimum of 1 year and a maximum of 2 years following vaccination.

\section{Patient Population}

Included were women diagnosed with R-UTIs who required medical consultation, study, and treatment at the Department of Urology of Villarrobledo General Hospital, Albacete, Spain. We used the criteria by Grabe et al. [1] to define UTI or R-UTI. We collected epidemiological data on our patients, such as age and any history of pathology and/or medical treatments (risk factors) that might be associated with the development of R-UTI. The only exclusion criteria were the presence of autoimmune disease or refusal of the patient to be treated with the vaccine.

The data collected from the patients' medical records were as follows: (a) number and frequency of UTIs; (b) duration and evolution of the R-UTI condition; (c) presence of risk factors for UTI (diabetes mellitus, urolithiasis, urinary incontinence, previous surgery for incontinence or prolapse of pelvic organs, permanent catheterization, intermittent catheterization, previous oncological or neurological history, or treatment with corticosteroids); and (d) results of general and urological physical examinations and urine cultures. The study period was from January 2013 to December 2016.

\section{Treatments}

In order to treat R-UTI, we employed sublingual vaccines that contained inactivated, whole bacteria. The vaccine consisted of 2 vials containing a suspension of $10^{9}$ inactivated, whole bacteria per millilitre; the prescriptions were individualized for each patient in terms of the desired bacterial composition of the vaccine based on the subject's previous urine cultures. The vaccine was then administered by means of 2 insufflations of spray into the sublingual mucosa once a day for 3 months.

Uromune $^{\circledR}$ is a commercially available bacterial vaccine (a named-patient preparation in Spain) produced by Inmunotek (Madrid, Spain) and marketed by Q-Pharma (Alicante, Spain). There are different types of formulation for this vaccine: (1) a generic formulation, consisting of 2 vials containing a suspension of $10^{9}$ inactivated whole bacteria per millilitre; the vaccine is a mixture of equal amounts of selected strains of Escherichia coli, Klebsiella pneumoniae, Proteus vulgaris, and Enterococcus faecalis; (2) a specific formulation, in which the prescriber selects the percentage of each bacterium to appear in the formulation; and (3) an autovaccine, composed of cultures of organisms taken directly from the patient's urine. We employed the specific formulation in this study, with a sublingual mucosal delivery route at a daily dose of 2 puffs of $100 \mu \mathrm{L}$ each $\left(10^{8}\right.$ bacteria/puff), avoiding the concomitant intake of food or beverage. The delivered dose was maintained under the tongue for a period of 1-2 min and then swallowed. The patients received this treatment for a period of 3 months. Each vaccine was formulated based on the presence of each bacterium in the previous urine cultures.

\section{Evaluation}

All patient data were included in the computerized medical records at our hospital. The following variables were collected retrospectively for at least 1 year before vaccination and prospectively for a minimum of 1 year and a maximum of 2 years after vaccination, in scheduled appointments: (a) number of recorded episodes of UTI; (b) number of urine cultures performed; and (c) type and number of packages of antibiotics consumed. All data were collected in terms of the absolute number of events during each monitoring period (before and after vaccination). Given that the preand post-vaccination periods represented different lengths of time, for purposes of comparison, the data were adjusted to the number of events per patient per year.

In our study, during the retrospective data collection period, we defined episodes of UTI as consultations with the primary care physician or at the emergency department due to UTI symptomatology, accompanied by a positive urine culture or dip stick. We evaluated the presence of symptoms and positive or negative urine cultures $3,6,9,12,18$, and 24 months after initiating the vaccine.

\section{Statistics}

Both descriptive and bivariate analyses were carried out. Linear and logistic regression models were constructed to explore which variables were associated with post-vaccination changes in the de- 
Table 1. Distribution of the number of positive cultures, episodes of urinary tract infection (UTI), and consumption of packages of antibiotics (per year) before vaccination according to medical risk factors $(n=166)$

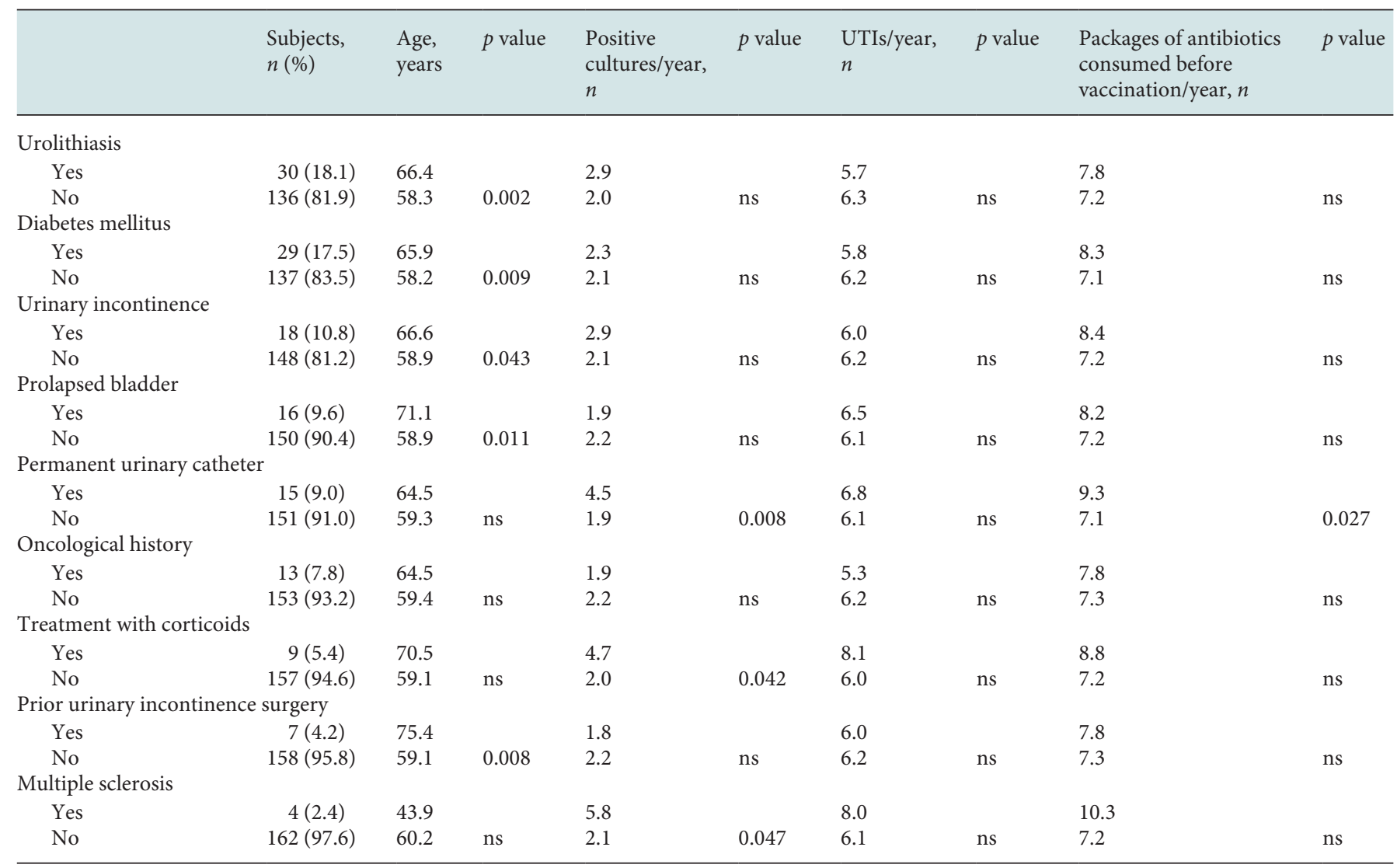

pendent variables, controlling the effect of confounding variables and assessing the contribution of each one of them. Finally, survival analyses were performed using the actuarial estimation and Kaplan-Meier methods to assess the probability of remaining UTIfree and maintaining negative urine cultures during the different time intervals studied after the initiation of vaccination. Subsequently, the survival curves were calculated, and comparisons were made using the Mantel-Haenszel log-rank test. The significance level used for all the tests in this study was an alpha error $\leq 5 \%$ $(p \leq 0.05)$. The statistical software used for data analysis was SPSS version 20 .

\section{Results}

\section{Clinical Profile}

A total of 166 women met the inclusion criteria for this study. The mean age of the participants was 62.3 years (SD 18.4, 95\% CI 59.4-65.1) with an age range between 19 and 89 years; $75.4 \%$ were older than 50 years. $53.6 \%$ of the patients did not present with risk factors related to the development of UTI. Amongst the remaining patients, a history of urinary urolithiasis (18.1\%) was the most frequently found predisposing factor. Table 1 shows the clinical risk factors associated with the development of R-UTI and the prevalence of each of these factors amongst our patients. Additionally, the following patient variables were presented in terms of their relationship to each risk factor: (1) age, (2) number of positive urine cultures per year before vaccination, (3) number of episodes of UTI per year before vaccination, and (3) number of packages of antibiotics consumed per year before vaccination. It is noteworthy that most of the risk factors were found amongst the older subjects; additionally, patients with permanent catheters, multiple sclerosis, or in treatment with corticoids had the highest number of positive cultures before vaccination. Paradoxically, there were no differences in pre-vaccination frequency of UTI according to the distinct risk factors.

\section{Safety}

In terms of side effects, only 2 episodes of mild glossitis were reported, which subsided spontaneously with- 
Table 2. Mean values $( \pm \mathrm{SD})$ of the number of events per patient per year analyzed before and after vaccination, and the percent decrease in these values after vaccination

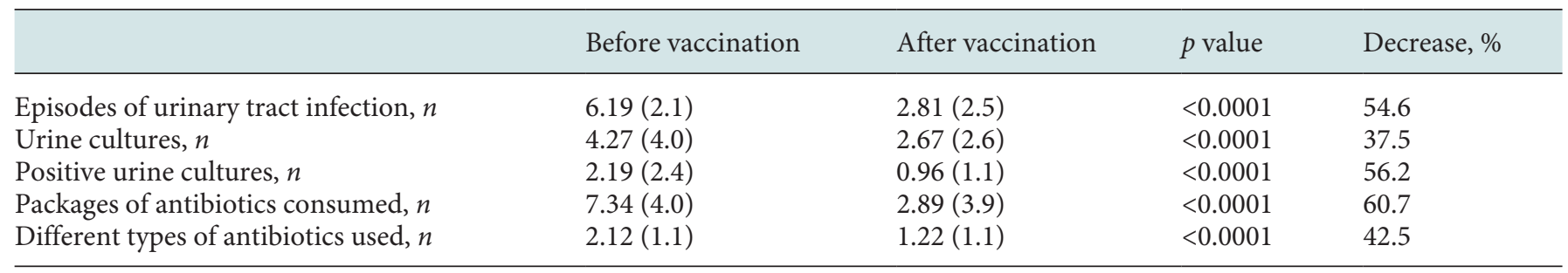

out the need to discontinue the vaccine. One patient experienced a flare-up of rheumatoid arthritis 7 days after initiating treatment with the sublingual vaccine and therefore discontinued the treatment. This patient was not included in the analysis.

\section{Analysis before Treatment with the Sublingual \\ Bacterial Vaccine}

The mean time of pre-treatment data collection was 2.7 years. During this pre-vaccination period, the patients reported an average of 6.2 episodes of UTI per year and an average number of 4.2 cultures per patient per year, of which an average of 2.2 per year were positive.

Of the 1,566 cultures reviewed, 811 were positive for different pathogens, of which the following were predominant: E. coli (74.8\%), K. pneumoniae (10.1\%), E. faecalis (5.7\%), Proteus mirabilis (2.3\%), and others (7\%). We reviewed the antibiograms of the 607 cultures that were positive for $E$. coli, and we were able to verify bacterial resistance to multiple antibiotics, the most frequent being to ampicillin (66.9\%), trimethoprim-sulfamethoxazole (46.9\%), cephalosporins of the first generation (34.3\%), quinolones (33.1\%), and amoxicillin/clavulanic acid (30.7\%). Of the clinical isolates of E. coli, $11.4 \%$ produced extended-spectrum $\beta$-lactamases. The average number of episodes of antibiotic resistance to $E$. coli per patient was 2.8. Each patient had consumed an average of 7.3 packages of antibiotics per year before vaccination. The most commonly used antibiotic was fosfomycin (31.2\%), followed by norfloxacin (16\%), amoxicillin-clavulanic acid (17.2\%), and ciprofloxacin (12.5); $94 \%$ of the patients had taken fosfomycin on occasion. The patients in our study had consumed an average of 2.1 different types of antibiotics per year in the pre-vaccination period.

\section{Analysis after Treatment with the Sublingual Bacterial Vaccine}

All of the patients included in the study completed the 3 -month vaccination treatment, and they were followed up for a minimum of 12 months and a maximum of 24 months after vaccination, with an average follow-up of 20 months. $81.9 \%$ of the women were followed up for more than 1 year and $57.8 \%$ were monitored for 2 years. There was a reduction of $54.6 \%$ in UTI episodes per year after vaccination (95\% CI 44.8-58.9). The average decrease in consumption of packages of antibiotics per year was $60.7 \%$ (95\% CI $46.0-63.3$ ), with a reduction of $42.5 \%$ in different types of antibiotic used.

$37.5 \%$ fewer urine cultures per patient per year were performed after vaccination (95\% CI 34.6-40.3), and the average decrease in the rate of positive urine cultures was $56.2 \%$. Table 2 shows the comparison between the results obtained before and those after vaccination. When differentiating between patients with and those without risk factors for R-UTI, the reduction in frequency of UTIs per year following vaccination was less marked amongst the women with risk factors than amongst the patients without risk factors ( 0.80 vs. 0.89 UTIs/year). This also occurred with respect to the number of positive urine cultures per year ( 0.77 vs. 0.82$)$.

\section{Evolutionary Analysis of the Patients}

The percentage of asymptomatic patients 3 months after initiating vaccination treatment was $74.4 \%$. The frequency of patients without UTI recurrence diminished during subsequent follow-ups, with $68.1 \%$ being without UTI recurrence at 6 months, $52.4 \%$ at 12 months, and $44.5 \%$ at 24 months. The cumulative probability of maintaining negative cultures after vaccination was $75 \%$ at 3 months, $37 \%$ at 12 months, and $18 \%$ at 24 months. The mean time during which patients maintained negative urine cultures after vaccination was higher amongst the women without a history of risk factors related to UTI ( $p=0.048)$, amongst the patients having had fewer than 3 episodes of $E$. coli resistance in previous urine cultures $(p=0.026)$, and amongst those whose infections were caused solely by $\operatorname{E}$. coli $(p<0.001)$ (Table 3). 
Table 3. Mean and median time (months) during which the patients maintained negative cultures after vaccination according to risk factors, the number of episodes of resistance to Escherichia coli, and the causal microbe(s)

\begin{tabular}{|c|c|c|c|c|c|}
\hline & Events & $\begin{array}{l}\text { Mean time, } \\
\text { months }\end{array}$ & $95 \% \mathrm{CI}$ & $\begin{array}{l}\text { Median time, } \\
\text { months }\end{array}$ & $p$ value \\
\hline Risk factors related to urinary tract infection & & & & & 0.048 \\
\hline Yes & $51 / 77$ & 11.2 & $9.1-13.2$ & 6 & \\
\hline Episodes of bacterial resistance & & & & & 0.026 \\
\hline$\leq 3$ & $54 / 104$ & 13.8 & $11.9-15.6$ & 12 & \\
\hline Causal bacteria for urinary tract infection & & & & & 0.001 \\
\hline Multimicrobial or not E. coli & $61 / 85$ & 10.4 & $8.6-12.2$ & 9 & \\
\hline E. coli & $34 / 78$ & 15.3 & $13.1-17.5$ & 18 & \\
\hline
\end{tabular}

Table 4. Variables associated with post-vaccination changes in the frequency of urinary tract infections (UTIs) and the frequency of positive cultures according to multivariate linear and logistic regression models

\begin{tabular}{|c|c|c|c|}
\hline & Coefficient $b$ & $95 \% \mathrm{CI}$ & $p$ value \\
\hline \multicolumn{4}{|l|}{ Changes in the frequency of UTIs } \\
\hline \multicolumn{4}{|l|}{ Linear regression model } \\
\hline Positive cultures before vaccination $(n)$ & 0.43 & 0.16 to 0.52 & $<0.0001$ \\
\hline Risk factors for UTI $(n)$ & -0.40 & -0.80 to -0.14 & 0.015 \\
\hline Subjective improvement after vaccination & 2.31 & 1.27 to 3.35 & $<0.0001$ \\
\hline \multirow[t]{2}{*}{ Constant } & 2.64 & 1.27 to 3.33 & $<0.0001$ \\
\hline & OR & $95 \% \mathrm{CI}$ & $p$ value \\
\hline \multicolumn{4}{|l|}{ Logistic regression model } \\
\hline Subjective improvement after vaccination & 1.15 & 1.084 to 2.079 & $<0.0001$ \\
\hline Positive cultures before vaccination $(n)$ & 24.4 & 7.29 to 82.45 & $<0.0001$ \\
\hline \multirow[t]{2}{*}{ Constant } & 2.104 & & $<0.0001$ \\
\hline & Coefficient $b$ & $95 \% \mathrm{CI}$ & $p$ value \\
\hline \multirow{2}{*}{\multicolumn{4}{|c|}{$\begin{array}{l}\text { Changes in the frequency of positive cultures } \\
\text { Linear regression model }\end{array}$}} \\
\hline & & & \\
\hline Positive cultures before vaccination $(n)$ & 0.92 & 0.87 to 0.97 & $<0.0001$ \\
\hline Subjective improvement after vaccination & 3.53 & 3.13 to 3.40 & $<0.0001$ \\
\hline \multirow[t]{2}{*}{ Constant } & -0.98 & -1.43 to -0.51 & $<0.0001$ \\
\hline & OR & $95 \% \mathrm{CI}$ & $p$ value \\
\hline \multicolumn{4}{|l|}{ Logistic regression model } \\
\hline Positive cultures before vaccination $(n)$ & 2.2 & 1.45 to 3.84 & 0.001 \\
\hline Subjective improvement after vaccination & 9.7 & 3.60 to 26.1 & $<0.0001$ \\
\hline Constant & 0.297 & & 0.019 \\
\hline
\end{tabular}

\section{Multivariate Analysis}

In the linear regression analysis, the variables associated with a greater reduction in the frequency of UTIs with vaccination were (a) a higher number of positive cul- tures before vaccination $(p<0.0001)$, (b) a lower number of risk factors for UTI $(p=0.015)$, and (c) having reported symptomatic improvement with vaccination $(p<$ $0.0001)\left(F=26.991 ; p<0.0001 ; R^{2}=0.403\right)$. Through lo- 
gistic regression, it was verified that for each episode of UTI prior to vaccination, the probability of improving this condition was multiplied by a factor of 1.15 ( $p<$ 0.0001 ), whereas amongst patients who experienced symptom improvement after vaccination, this probability was multiplied by 24 ( $p<0.0001$; Nagelkerke $\left.R^{2} 0.40\right)(\mathrm{Ta}-$ ble 4).

Furthermore, the variables associated with a greater reduction in the frequency of positive cultures after vaccination were (a) a higher number of positive cultures before vaccination $(p<0.0001)$ and (b) having reported symptomatic improvement $(p<0.0001 ; F=431.16 ; p<$ $0.0001 ; R^{2}=0.889$ ). Through logistic regression, we found that for each positive culture prior to vaccination, the probability of reversing this result with vaccination was multiplied by a factor of 2.3 ( $p=0.001$ ), and by 9.7 for the women with symptomatic improvement following vaccination $\left(p<0.0001\right.$; Nagelkerke $\left.R^{2} 0.30\right)$ (Table 4$)$.

\section{Discussion and Conclusion}

UTIs are amongst the most prevalent infectious diseases, and the economic burden they impose on society is considerable. Therefore, the concern with finding an effective treatment for recurrent UTI without the side effects and bacterial resistance caused by antibiotic prophylaxis $[7,8]$ is justified. The launch of the first oral vaccine for prevention of repeat UTI, namely, the immunotherapeutic formulation OM-89 (marketed in Europe by EurimPharm GmbH as Uro-Vaxom ${ }^{\circledR}$ ), was in the late 1990s [9]; it is a bacterial extract prepared from 18 strains of $E$. coli [10]. This oral vaccine in the form of a capsule is normally administered as a daily dose for 3 months and is recommended by the European Association of Urology for women with recurrent, uncomplicated UTI (GCC: 1a, GR: B) [11]. Subsequently, a spray-type vaccine emerged for the prophylaxis of recurrent UTI, with sublingual administration.

There is an important difference between the immunotherapeutic formulation OM-89 (Uro-Vaxom ${ }^{\circledR}$ ) and the sublingual formulations that are currently gaining more prominence on the market (Uromune ${ }^{\circledR}$ ); that is to say, the first vaccine (Uro-Vaxom ${ }^{\circledR}$ ) originates from lysed bacteria, whereas sublingual "spray-type" formulations contain whole, inactivated bacteria instead. The sublingual forms of the vaccine may provide greater clinical benefits, since whole, inactivated bacteria present themselves in a more natural way to the innate immune system, offering their whole potential [12].
The sublingual epithelium contains a dense network of dendritic cells that play an essential role in linking innate and adaptive immune responses [13]. Recent studies of the immunological mechanisms underlying the clinical benefits of Uromune ${ }^{\circledR}$ in the treatment of R-UTI have shown that sublingual vaccines stimulate human dendritic cells, with the ability to generate Th1, Th17, and IL10-producing T cells [14].

Regarding the efficacy of sublingual immunostimulant compounds for the prevention of recurrent UTI, Uromune $^{\circledR}$ (a sublingual preparation of E. coli, K. pneumoniae, $P$. vulgaris, and E. faecalis) was recently evaluated in a multicentre, retrospective observational study in Spain [15]. Women with a history of recurrent UTI who received daily prophylaxis with Uromune ${ }^{\circledR}$ for 3 months had significantly fewer recurrences over a 15-month period than women who received trimethoprim-sulfamethoxazole daily for 6 months, with a $75 \%$ improvement in the number of UTIs in the group treated with Uromune ${ }^{\circledR}$.

The mean age of the patients in our study was 62.3 years, and $62.1 \%$ of them were over the age of 60 years. This more advanced average age, which is a limitation to our study, reflects the profile of patients who most commonly attend our urology clinic, given that younger patients, whose infections are less resistant and more responsive to antibiotic therapy, are usually successfully treated in primary care. For example, in a 2018 study of 75 women treated with Uromune ${ }^{\circledR}$ in the UK, most of the patients who experienced recurrences after treatment with the vaccine were postmenopausal. Concretely, of the 50 postmenopausal women in the UK study, 14 experienced posttreatment infection, signifying that Uromune ${ }^{\circledR}$ successfully prevented UTI recurrence in $72 \%$ of these patients. However, only 2 of the 25 premenopausal women treated with the vaccine experienced recurrence, indicating that Uromune $^{\circledR}$ effectively prevented new recurrences of UTI for $88 \%$ of the premenopausal women [16].

The previously mentioned Spanish study comparing R-UTI patients treated with sublingual bacterial immunotherapy to those given trimethoprim-sulfamethoxazole [15] excluded subjects with a personal history of risk factors for R-UTI (urolithiasis, catheters, immunosuppression, etc.). In the present study, these patients were not excluded, since we considered that the percentage of R-UTI patients with risk factors related to UTI was very elevated, and therefore the possible benefits from the vaccine should be evaluated for these women as well.

We established a minimum follow-up period of 1 year and a maximum of 2 years after initiating treatment, due 
to the fact that existing studies on sublingual bacterial vaccines do not provide information beyond the first year following treatment. Moreover, since R-UTI tends to be a chronic pathology, it is important to understand patient evolution beyond a 1-year follow-up.

The rate of patients without UTI recurrence and presenting negative urine cultures decreased progressively throughout the follow-up period, likely due to loss of protective antibodies. This leads us to consider revaccinating patients whose symptoms reappear after initial success with the treatment.

A study that included 699 women with R-UTI showed that Uromune ${ }^{\circledR}$ has an immunological memory; that is to say, of the group of patients treated for 3 months with the sublingual bacterial vaccine, $90.3 \%$ remained UTI-free after 1 year of follow-up [17].

In the current study, the patients who showed the greatest improvement in their UTI symptoms and remained free of positive urine cultures for the longest time after vaccination were (1) patients with no history of risk factors related to the development of UTI; (2) patients who presented with pre-vaccination urinary infections caused only by E. coli; and (3) patients who had $\leq 3$ episodes of $E$. coli resistance in urine cultures before vaccination.

As discussed earlier, the patient sample we selected is one of the principle limitations to this study, in terms of the subjects' advanced age and the inclusion of patients with a history of risk factors for developing UTI. Although the inclusion of patients with risk factors for UTI may be considered a limitation to our study, we took this variable into account in our analysis and compared outcomes between the women with and those without risk factors. Furthermore, our study is not a randomized clinical trial. The greatest disadvantage of a pretest-posttest quasi-experimental design is the lack of a control group, which limits the establishment of correlations between results and the treatment under evaluation. However, results from non-randomized clinical trials such as ours can still be considered valid, although to a lesser degree.

In conclusion, the use of a sublingual bacterial vaccine in preventing UTI in women is an effective and safe treatment that produces a significant reduction in symptoms, lengthens the disease-free period, and contributes to a decrease in the number of recurrences. Given the high rates of antibiotic resistance amongst patients with R-UTI, the sublingual vaccine may be useful in decreasing the number of antibiotic prescriptions and therefore improving the problem of antibiotic multiresistance.

\section{Acknowledgements}

The authors have not had any funding for this study and are responsible for the study design, data collection and analysis, the decision to publish, and the preparation of the manuscript.

\section{Statement of Ethics}

All subjects have given written informed consent. The reasons why we do not attach an ethics committee reference number are that the sublingual bacterial vaccine used in our study is Uromune ${ }^{\circledR}$, a drug approved by the authorities of our country (Spanish Agency of Medicines and Medical Devices) for the prevention of R-UTI. In addition, as it was a study of clinical practice results, no approval was requested from the centre's ethics committee, only the informed consent of the patients. Our project included patients from January 2013, months before the Declaration of Helsinki (Fortaleza, Brazil, October 2013), in which all research studies that included human subjects had to register in a public access database before recruiting the first subject.

\section{Disclosure Statement}

The authors have no conflicts of interest to declare.

\section{Author Contributions}

P.C.-L.: design of the study, data collection, patient selection, data analysis, and manuscript realization; J.M.-R.: design of the study, data collection, and patient selection; L.L.-G.: data collection and patient selection; J.M.G.-B.: statistical analysis of the data; H.P.-N.: data collection; A.S.S.-S.: design of the study and statistical analysis of the data.

\section{References}

1 Grabe M, Johansen TEB, Botto H, Cai T, Çek $\mathrm{M}$, Köves B, et al. Guidelines on urological infections. Arnhem: European Association of Urology; 2015.

2 Geerlings SE, Beerepoot MA, Prins JM. Prevention of recurrent urinary tract infections in women: antimicrobial and nonantimicrobial strategies. Infect Dis Clin North Am. 2014 Mar;28(1):135-47.

3 Jepson RG, Williams G, Craig JC. Cranberries for preventing urinary tract infections. Cochrane Database Syst Rev. 2012 Oct; 10(1):CD001321.

4 De Leo V, Cappelli V, Massaro MG, Tosti C, Morgante G. Evaluation of the effects of a natural dietary supplement with cranberry, Noxamicina ${ }^{\circledR}$ and D-mannose in recurrent urinary infections in perimenopausal women [in Italian]. Minerva Ginecol. 2017 Aug; 69(4):336-41. 
5 Holmgren J, Czerkinsky C. Mucosal immunity and vaccines. Nat Med. 2005 Apr;11(4 Suppl):S45-53.

6 Cuburu N, Kweon MN, Song JH, Hervouet C, Luci C, Sun JB, et al. Sublingual immunization induces broad-based systemic and mucosal immune responses in mice. Vaccine. 2007 Dec;25(51):8598-610.

7 Nelson CP, Hoberman A, Shaikh N, Keren R, Mathews R, Greenfield SP, et al. Antimicrobial Resistance and Urinary Tract Infection Recurrence. Pediatrics. 2016 Apr;137(4): e20152490.

8 Beerepoot MA, Geerlings SE, van Haarst EP, van Charante NM, ter Riet G. Nonantibiotic prophylaxis for recurrent urinary tract infections: a systematic review and meta-analysis of randomized controlled trials. J Urol. 2013 Dec;190(6):1981-9.

9 Tammen H; The German Urinary Tract Infection Study Group. Immunobiotherapy with Uro-Vaxom in recurrent urinary tract infection. Br J Urol. 1990 Jan;65(1):6-9.
10 Bauer HW, Alloussi S, Egger G, Blümlein HM, Cozma G, Schulman CC; Multicenter UTI Study Group. A long-term, multicenter, double-blind study of an Escherichia coli extract (OM-89) in female patients with recurrent urinary tract infections. Eur Urol. 2005 Apr;47(4):542-8.

11 Grabe M, Bartoletti R, Johansen TEB, Botto $\mathrm{H}$, Çek M, Naberet KG, et al. Guidelines on urological infections. Arnhem: European Association of Urology; 2010.

12 Oyston P, Robinson K. The current challenges for vaccine development. J Med Microbiol. 2012 Jul;61(Pt 7):889-94.

13 Czerkinsky C, Cuburu N, Kweon MN, Anjuere F, Holmgren J. Sublingual vaccination. Hum Vaccin. 2011 Jan;7(1):110-4.

14 Benito-Villalvilla C, Cirauqui C, Diez-Rivero CM, Casanovas M, Subiza JL, Palomares O. MV140, a sublingual polyvalent bacterial preparation to treat recurrent urinary tract infections, licenses human dendritic cells for generating Th1, Th17, and IL-10 responses via Syk and MyD88. Mucosal Immunol. 2017 Jul;10(4):924-35.
15 Lorenzo-Gómez MF, Padilla-Fernández B, García-Criado FJ, Mirón-Canelo JA, Gil-Vicente A, Nieto-Huertos A, et al. Evaluation of a therapeutic vaccine for the prevention of recurrent urinary tract infections versus prophylactic treatment with antibiotics. Int Urogynecol J Pelvic Floor Dysfunct. 2013 Jan; 24(1):127-34.

16 Yang B, Foley S. First experience in the UK of treating women with recurrent urinary tract infections with the bacterial vaccine Uromune $^{\circledR}$. BJU Int. 2018 Feb;121(2):28992.

17 Lorenzo-Gómez MF, Padilla-Fernández B, García-Cenador MB, Virseda-Rodríguez ÁJ, Martín-García I, Sánchez-Escudero A, et al. Comparison of sublingual therapeutic vaccine with antibiotics for the prophylaxis of recurrent urinary tract infections. Front Cell Infect Microbiol. 2015 Jun;5:50. 